\title{
Contribution of Neuropeptides and Neurotransmitters in colitis
}

\section{Cezary Skobowiat}

Department of Clinical Physiology, University of Warmia and Mazury, 10-718 Olsztyn, Poland

Department of Pathology, University of Tennessee Health Science Center, Memphis, TN 38163, USA

\begin{abstract}
Abbreviations: ANS: Autonomic Nervous System; ENS: Enteric Nervous System; SChG: Sympathetic Chain Ganglia; IMG: Inferior Mesenteric Ganglion; CD: Crohn's Disease; UC: Ulcerative Colitis; IBD: Inflammatory Bowel Disease; IBS: Irritable Bowel Syndrome; CRH: Corticotropin-Releasing Hormone; NA: Noradrenaline; NPY: Neuropeptide Y; NO: Nitric oxide; NOS: Nitric oxide Synthase; SP: Substance P; CGRP: Calcitonin Gene-Related Peptide; VIP: Vasoactive Intestinal Peptide; 5-HT: Serotonin; CART: Cocaine and Amphetamine Regulated Transcript
\end{abstract}

\section{Introduction}

Colitis is a chronic, relapsing, and remitting disease involving complex interactions between genes, immune system, and neuroendocrine feedback. Important advances have been recently made to support the awareness that neuroendocrine factors can significantly impact the immune response in the gut [1]. Unlike the other nervous systems of the body, the enteric nervous system (ENS) can work without central input from the brain and is often considered as "the brain-in-the gut"[2]. However, both the ENS and the central nervous system (CNS) can amplify or modulate aspects of intestinal inflammation through secretion of neuropeptides and non-peptide neurotransmitters that serve as a link between the ENS and CNS. Neuropeptides are defined as any peptide released from the nervous system that serves as an intercellular signaling molecule [3]. Neuropeptides and other neurotransmitters, when released at the nerve endings in the colon side, diffuse into surrounding tissues and bind to their corresponding receptors affecting peristalsis, fluid secretion, and digestive processes $[3,4]$. Locally released neurotransmitters act also directly on contiguous vasculature, mast cells and muscles, prompting their release of proinflammatory factors $[5,6]$. Immune cells localized in the colon wall express various neurotransmitter receptors, and they produce cytokines and other immune/inflammatory mediators like chemokines and free radicals. Once the neurotransmitter has reached targeted cells and occupied appropriate receptors the initiated signal transduction pathways of cytokine production has been started [7]. On the other hand, regulatory cytokines released on colon wall can bind to specific receptors localized on sensory nerve fibers and trigger neuronal response. This bidirectional cross-talk between neuronal and immune factors is crucial to maintenance the visceral homeostasis and also plays an important role during colitis. Neuropeptides thought to play key roles in colitis include substance $\mathrm{P}(\mathrm{SP})$, calcitonine gene-related peptide (CGRP), neuropeptide Y (NPY), corticotropinreleasing hormone $(\mathrm{CRH})$, vasoactive intestinal peptide (VIP), galanin (GAL) and cocaine and amphetamine regulated transcript (CART). Furthermore, neurotransmitters like noradrenaline (NA), serotonin (5-HT), and gaseous nitric oxide (NO). All above mentioned substances can control a variety of functions within the gastrointestinal (GI) tract under physiological and pathological conditions [8]. Neurotransmitters may evoke remarkably different and even opposing effects depending on the concentration of released neurotransmitter/ co-transmitters, receptor expression levels, affinity, and timing of neurosecretory activity in relation to the inflammatory course [4,9]. The enhanced neuropeptide release has been reported in the colon during inflammation accompanying ulcerative colitis (UC), Crohn disease (CD) and irritable bowel syndrome (IBS) (Figure 1).

\section{Substance $\mathrm{P}$ (SP) and calcitonin gene-related peptide (CGRP)}

Sensory innervation of colon comprise of intrinsic primary afferent neurons (IPANs), whose cell bodies are located in the myenteric or submucosal plexuses and extrinsic primary afferent neurons (EPANs), with their somata in the dorsal root ganglia (DRG). These neuronal populations contain mainly neuropeptides, such as SP and CGRP, stored in vesicles that are released upon depolarization. SP- and CGRPpositive DRG neurons often co-express transient receptor potential vanilloid (TRPV1) channel. TRPV1 is one of the key components of nociceptive signal transduction pathways and is widely distributed throughout the GI tract as well. In experimentally induced colitis in rats activation of TRPV1 nociceptive afferent nerve terminals, lead to enhanced release of CGRP and SP not only at the inflammation side but also in adjacent visceral organs [10]. The liberation of CGRP and SP results in vasodilatation, plasma extravasation, and leukocyte migration, which is commonly referred to as neurogenic inflammation

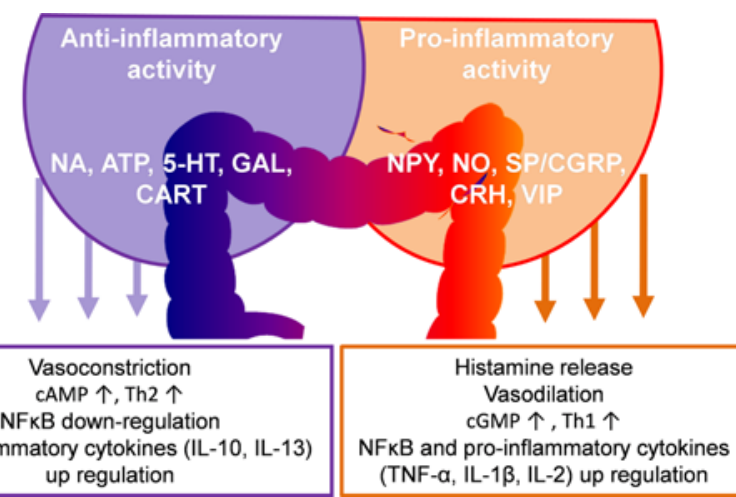

Figure 1: The contribution of particular neurotransmitters in colonic homeostasis. The homeostasis of colon activity is regulated by vasoconstrictory stimuli, including CAMP, anti-inflammatory cytokines, inhibition of NF-KB and Th-2 mode of immune response (left side of figure) and vasodilatory stimuli, including CGMP, pro-inflammatory cytokines, activation of NF-KB and Th-1 mode of immune response (right side of the figure).

Corresponding author: Cezary Skobowiat PhD, DVM, Department of Clinica Physiology, University of Warmia and Mazury, 10-718 Olsztyn, Poland and Department of Pathology, University of Tennessee Health Science Center Memphis, TN 38163, USA, E-mail: cskobowi@uthsc.edu

Received October 15, 2011; Accepted November 14, 2011; Published November 21, 2011

Citation: Skobowiat C (2011) Contribution of Neuropeptides and Neurotransmitters in colitis. J Veterinar Sci Technol S5:001. doi:10.4172/2157-7579.S5-001

Copyright: (c) 2011 Skobowiat C. This is an open-access article distributed under the terms of the Creative Commons Attribution License, which permits unrestricted use, distribution, and reproduction in any medium, provided the original author and source are credited. 
and is related to the axon reflex phenomenon. SP and CGRP stimulate T-cell migration, induce secretion of the proinflammatory cytokines like IL- $1 \beta$, interferon gamma (IFN- $\gamma$ ), and tumor necrosis factor alpha (TNF-a) [9]. The molecular mechanism underlying this inflammatory response involves, in part, the activation of the nuclear transcription factor $(\mathrm{NF}-\mathrm{\kappa B})$ system and the consequent inflammatory cascade [11]. In conclusion, the role of sensory neuronal activation and consecutive neuropeptide release with respect to protection or aggression in colonic inflammation seems to remain a double-edged sword. For example, release of SP/CGRP from afferent terminals triggers mRNA up-regulation followed by secondary increase in the release of SP/ CGRP in the colon at later time [10].

\section{Noradrenaline (NA)}

The sympathetic nervous system (SNS), with its main neurotransmitter NA, which is released from postganglionic varicosities innervating the peripheral lymphoid organs, provides the primary pathway for the neural regulation of immune function [6,7]. There is ample evidence that the immunomodulatory effect of NA is mediated through cAMP. NA and the adrenergic agonists may influence the immune response directly, through adrenergic $\beta$-receptors expressed on macrophages and other immunologically competent cells, as well as indirectly via alteration of endogenous NA levels by influencing the activity of release-regulating presynaptic $\alpha 2$-adrenoceptors located on sympathetic nerve terminals. Activation of latter receptors results in a negative feedback effect on NA release, leading to decreased extracellular NA concentration. On the other hand, activation of neurotransmitter receptors that stimulate adenylate-cyclase leads to a shift toward $\mathrm{T}$ helper 2 (Th2)-type responses, which are both neuroprotective and anti-inflammatory, whereas down regulation of intracellular cAMP stimulates a T helper 1 (Th1)-type response, resulting in cell destructive effects and inflammation [7]. Prior studies have shown that the loss of noradrenergic fiber density during colitis is due to inhibition of N-type voltage-gated $\mathrm{Ca}^{2+}$ current in postganglionic sympathetic neurons $[12,13]$. Furthermore, during experimental colitis, the axotomy of sympathetic nervii supplying the descending colon in pigs are showed to decrease in the number of catecholamine-containing perikarya localized in sympathetic chain ganglia (SChG) and inferior mesenteric ganglion (IMG) - the main sources of sympathetic innervation of the colon $[14,15]$. These findings provide evidence that inflammation at colon wall influences the chemical coding of sympathetic neurons affecting neuronal plasticity.

Interest in the role of the SNS under inflammatory bowel disease (IBD) is rapidly increasing. However, much work needs to be done to enhance the understanding of how SNS function is altered during IBD and what contribution, if any, these changes make to pathogenesis [12]. One of the sympathetic co-transmitters, ATP, is also engaged during colitis, namely by the reduced purinergic transmission to submucosal arterioles that was noticed, and may be due to increased degradation of ATP throughout colitis [16]. On the other hand, venlafaxine, an 5-HT/NA reuptake inhibitor, alters colonic compliance and tone and tends to reduce sensation during colonic distention in healthy humans, showing usefulness in colonic disorders affecting motor and possibly sensory functions $[17,18]$.

\section{Neuropeptide Y (NPY)}

NPY has been shown to elicit diverse biological functions including hypothalamic control of food intake, anxiety and sedation. This polypeptide is attached to heptahelical G-protein coupled receptors, which are widely distributed in the ENS and CNS. It has been found that NPY expression is up regulated in enteric neurons during experimental colitis in mice [19]. Thus, NPY is an inducible gene in enteric neurons that can promote inflammation. It has been demonstrated that NPY increases the number of NOS (nitric oxide synthase)-producing neurons in the murine ENS ganglia what can affect intestine vascularization and motility [20]. The administration of NPY antisense oligodeoxynucleotides (ODNs) in rats leads to an amelioration of experimentally-induced colitis, suggesting that NPY plays an important role in modulating of inflammation throughout colitis. Therefore, the NPY antisense ODNs may be a useful therapeutic approach to the treatment of ulcerative colitis (UC) and other intestinal diseases with inflammatory signs [21].

\section{Nitric Oxide (NO)}

$\mathrm{NO}$ is a gaseous mediator that exerts key regulatory functions in mammalian cells. Low levels of NO play homeostatic functions and counteract inflammation, whereas high amounts of $\mathrm{NO}$ cause tissue destruction and cellular death. NOS generates nitric oxide (NO), a major non-adrenergic non-cholinergic (NANC) neurotransmitter, which mediates relaxation responses of smooth muscle cells in the GI tract. NO has been identified to play a role in a variety of enteric neuropathies like Crohn's disease (CD), UC, Chagas disease, diabetic gastroparesis, achalasia and pyloric stenosis [20]. Inflammatory cytokines and infiltrating immune cells appear to be responsible for much of the colonic oxidative stress that is a hallmark of IBD and colonic nitrite levels serve as a sensitive marker of disease activity in colitis. When produced in small amounts, NO generally exerts positive effects in the gastrointestinal tract. However, under inflammatory colonic conditions, greatly increased NO levels have been reported, for example, in human IBD and in animal models of colitis [22]. It has been demonstrated that increased NO levels in the colon during UC is derived mostly from myenteric neurons than from epithelium [20]. Vascular resistance accompanying colitis is associated with higher levels of vasodilating $\mathrm{NO}$ in rat colonic mucosa [22]. Cytokines release during $\mathrm{UC}$ in rats induced expression of iNOS leading to a steep rise of NO synthesis [23]. In a rodent model of colitis, the new NO antagonists (NI-NOD1 and NI-NOD2) potently decreased inflammation. These data show that NI-NODs are effective in both in vitro and in vivo models of inflammation, mimicking the positive effects of low levels of NO and suppressing NOS-induced NO production [24].

\section{Corticotropin-releasing hormone (CRH)}

$\mathrm{CRH}$ is a 41 -amino acid hypothalamic peptide that modulates the synthesis and release of adrenocorticotropic hormone (ACTH) from the pituitary, leading to release of anti-inflammatory corticosteroids from the adrenal glands. A major function of $\mathrm{CRH}$ is to coordinate the endocrine, behavioral, immune, and visceral responses to stress [25]. Emerging evidence also links activation of the CRH-dependent signaling pathways with modulation of intestinal inflammation [26] There are functional differences between CRH receptor 1 (CRH-R1) and CRH-R2 activation. R1 stimulation evokes colonic motility, causes proinflammatory response with enhanced visceral nociception whereas R2 activation inhibits gastric emptying, reduces visceral perception and provokes anti-inflammatory changes reviewed in [27] CRH-R1 agonists promote intestinal inflammation, as well as endogenous inflammatory angiogenesis whereas CRH-R2 ones attenuate these features [28]. The enhanced expression of CRH and $c$-fos inside the hypothalamus (paraventricular nucleus) during experimentally induced acute colitis in rats has also been observed. This feature is probably evoked by the stimulatory effects of pro-inflammatory 
cytokines on visceral sensory afferents what enhances the excitability and activity of the hypothalamus pituitary adrenal (HPA) axis [29,30]. In contrary to the anti-inflammatory effects of hypothalamic $\mathrm{CRH}$ through induction of glucocorticoids, CRH secreted peripherally by immune cells, nerve fibers, and possibly additional cell types may act locally as a proinflammatory mediator [31]. In a chronic colitis model in rats the over expression of CRH-RI has been noticed [32]. The worsening of colitis symptoms occurred during periods of emotional stress suggests that IBS may be, at least partially, the result of the central $\mathrm{CRH}$ excess [30]. IBS in human lead to a mild elevation of plasma cortisol and significantly blunted ACTH levels suggest a deregulation of the HPA axis [33]. As most recently shown, gut [32], similarly to skin [34] and joints [35], has formed a local equivalent of the HPA axis which contributes to the central one and modulates its activity.

\section{Vasoactive intestinal peptide (VIP)}

VIP is a potent neuroendocrine mediator of diverse physiological responses, and is expressed prominently in primary immune organs and neurons of ENS and CNS [36]. VIP inhibits inflammatory pathways by reducing production of TNF- $\alpha$, IL- 6 and IL-12. Other studies have shown that VIP enhances the differentiation of $\mathrm{T}$ helper cells and promotes release of histamine by mast cells, thereby inducing erythema [37]. Normal activity of VIP transmission is critical in maintaining the intestinal immune homeostasis and selective enhancement of VIP signaling activates cAMP/PKA signal transduction pathways sufficiently to enhance colitis in mice. Taken together these and other findings suggest that VIP may manifest either an inhibitory or stimulatory effect on immune and immunopathological reactions in intestine [38].

\section{Galanin (GAL)}

In humans, GAL is a 30 -aa-long neuroendocrine peptide, for which its physiological functions are regulated by $\mathrm{G}$ protein-coupled receptors. GAL is found throughout the CNS and ENS, and its effects include a control of memory acquisition, modulation of appetite or sexual conduct, GI motion as well as effects on nociception during colitis. Both, colitis and the axotomy of colon wall cause an increase in GAL-immunoreactivity in the autonomic nervous system [14,15] and ENS in pigs [39]. Treatment with GAL is able to reduce the severity of the colitis by lowering the incidence of diarrhea, weight loss, infiltration of the inflammatory cells, mucosa disruption and edema [40]. GAL treatment has a significant preventive effect in TNBS-induced acute model of colitis in rats [41].

\section{Serotonin (5-HT)}

5-HT is a monoamine neurotransmitter that is classically recognized for its functions in the CNS, where it plays important roles in regulating mood, body temperature, sleep, sexuality, appetite, and metabolism. The vast majority of 5-HT (approximately 95\%) is localized to the intestine, where is synthesized by serotoninergic neurons of ENS as well as in the enterochromaffin (EC) cells [42]. As a paracrine factor, 5-HT targets mucosal projections of intrinsic primary afferent neurons to initiate enteric peristaltic and secretory reflexes. 5-HT is likely to play a role in mucosal homeostasis. Mucosal 5-HT modulates the immune response, and thus, is able potentially influence intestinal inflammation. 5-HT has been shown to promote lymphocyte activation and secretion proinflammatory cytokines [43]. Moreover, dendritic cells, lymphocytes, macrophages, endothelial cells, and enteric epithelial cells all express 5-HT receptors. Additionally, activation of specific signaling molecules of the NF- $\mathrm{kB}$ pathway is mediated by 5 -HT during intestinal inflammation [42]. Findings of a recent studies have provided a new mechanistic insights into antiinflammatory and immunosuppressive activities of 5-HT3 receptor antagonist (tropisetron) during rat IBD [44].

\section{Cocaine and amphetamine regulated transcript (CART)}

CART was originally given a role in drug abuse but the current focus on CART-induced effects is feeding behavior and body weight regulation as well the neuromodulatory and/or neurotrophic effects in intestine [45]. The coexistence of CART with NOS and VIP in the same neural population inside GI indicates that this peptide may influence NO- and VIP-induced effects during colitis [46]. CART is also implicated in promoting the survival of animal ENS neurons and in the protection of enteric neurons in intestinal disorders, neuronal stress or injury $[47,48]$. Other study suggests that CART influences on GI function are mostly via $\mathrm{CRH}$-dependent mechanism and peripheral cholinergic pathways [49]. Further examinations need to be undertaken to elicit the valuableness of CART in intestinal functions especially during colitis (Figure 2).

\section{Conclusion}

Colitis is a chronic multifactorial disease that targets the colon and as of recently afflicts more often the western population exacts a substantial toll on the economy with loss of productivity from disabilities and cost of medication. Despite advances in understanding of colitis pathogenesis and discovery of new treatments, the patients continue to suffer from this refractory disease [3]. Current treatment of colitis is expensive (antagonists of TNF- $\alpha$; adalimumab, infliximab, certolizumab) and frequently toxic (5-aminosalicylates) [50]. Furthermore, they are not effective in all patients [51]. The inadequacy of conventional therapy has motivated investigators to develop a novel approach to treat colitis. For example, tachykinin NK receptors inhibitors [52,53] or natural products which inhibit the NF- $\kappa B$ activity [54] have been recently under intense investigation as the newest target for colitis treatment.

Colitis is an extremely complex illness involving multiple levels of interaction between the neural, immune, and endocrine systems. The close anatomical and functional association between nerves, lymphoid organs, and intestinal cells suggests multidirectional cross-talk between them, where neuropeptides and cytokines act as viable neuroendocrine

G-protein coupled receptor

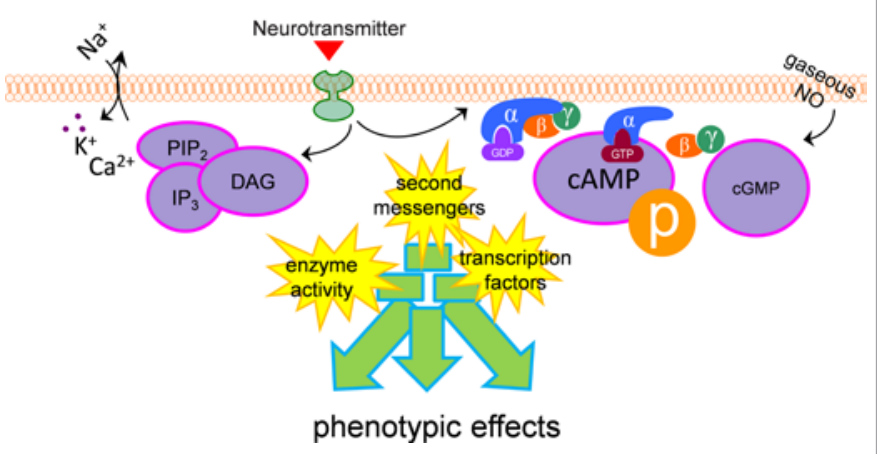

Figure 2: Molecular pathways of neurotransmitters actions in colon wall. Various intracellular pathways are active in the enterocytes (summarized in this figure) and lead to plethora of phenotypic effects in those cells (described in more detail in the text), that depend on the kind of neurotransmitter affecting the cell. 
linkage between these systems. However, the complexity of interactions between neuropeptides, conflicting study results, and opposing mechanisms of action of the neuropeptides, discussed warrants research in this field. Further clarification of the molecular mechanisms of neuropeptides and their effects on human diseases may yield treatment options in the future. This review highlights some aspects of bidirectional co-operation of neuro-immune factors and their impact on etiopathogenesis and course of colitis, mainly in human and laboratory animals. However, data obtained from these animal models cannot be directly extra pooled to human regarding evident differences in colon anatomy and physiology between two. The promising prospective alternatives constitute experiments performed on pigs $[14,39,55]$.

\section{Acknowledgments}

The author appreciates help of Dr. Tiffany Seagroves, Dr. Zorica Janjetovic and Dr. Blazej Zbytek.

\section{References}

1. Mawdsley JE, Rampton DS (2006) The role of psychological stress in inflammatory bowel disease. Neuroimmunomodulation 13: 327-336.

2. Furness JB (2006) Novel gut afferents: Intrinsic afferent neurons and intestinofugal neurons. Auton Neurosci 125:81-85.

3. Gross KJ, Pothoulakis C (2007) Role of neuropeptides in inflammatory bowel disease. Inflamm Bowel Dis 13: 918-932.

4. Engel MA, Becker C, Reeh PW, Neurath MF (2011) Role of sensory neurons in colitis: increasing evidence for a neuroimmune link in the gut. Inflamm Bowel Dis 17:1030-1033.

5. Anton PA, Shanahan $F(1998)$ Neuroimmunomodulation in inflammatory bowel disease. How far from "bench" to "bedside"? Ann N Y Acad Sci 840: 723-734.

6. Takami Y, Mantyh CR, Pappas TN, Takahashi T, Koda K et al. (2009) Extrinsic surgical denervation ameliorates TNBS-induced colitis in rats. Hepatogastroenterology 56: 682-686.

7. Szelenyi J, Vizi ES (2007) The catecholamine cytokine balance: interaction between the brain and the immune system. Ann N Y Acad Sci 1113: 311-324.

8. Magro F, Vieira-Coelho MA, Fraga S, Serrao MP, Veloso FT et al. (2002) Impaired synthesis or cellular storage of norepinephrine, dopamine, and 5-hydroxytryptamine in human inflammatory bowel disease. Dig Dis Sci 47: 216-224.

9. Johansson M, Jonsson M, Norrgard O, Forsgren S (2008) New aspects concerning ulcerative colitis and colonic carcinoma: analysis of levels of neuropeptides, neurotrophins, and TNFalpha/TNF receptor in plasma and mucosa in parallel with histological evaluation of the intestine. Inflamm Bowel Dis $14: 1331-1340$

10. Pan XQ, Gonzalez JA, Chang S, Chacko S, Wein AJ et al. (2010) Experimental colitis triggers the release of substance $P$ and calcitonin gene-related peptide in the urinary bladder via TRPV1 signaling pathways. Exp Neurol 225: 262-273.

11. Ursino MG, Vasina V, De Ponti F (2009) Protection from DNBS-induced colitis by the tachykinin NK(1) receptor antagonist SR140333 in rats. Eur J Pharmaco 603: 133-137.

12. Boisse L, Chisholm SP, Lukewich MK, Lomax AE (2009) Clinical and experimental evidence of sympathetic neural dysfunction during inflammatory bowel disease. Clin Exp Pharmacol Physiol 36: 1026-1033.

13. Motagally MA, Neshat S, Lomax AE (2009) Inhibition of sympathetic N-type voltage-gated $\mathrm{Ca}^{2+}$ current underlies the reduction in norepinephrine release during colitis. Am J Physiol Gastrointest Liver Physiol 296: G1077-1084.

14. Skobowiat C, Gonkowski S, Calka J (2010) Phenotyping of sympathetic chain ganglia (SChG) neurons in porcine colitis. J Vet Med Sci 72: 1269-1274.

15. Skobowiat C, Calka J, Majewski M (2011) Axotomy induced changes in neuronal plasticity of sympathetic chain ganglia $(\mathrm{SChG})$ neurons supplying descending colon in the pig. Exp Mol Pathol 90: 13-18.

16. Lomax AE, O'Reilly M, Neshat S, Vanner SJ (2007) Sympathetic vasoconstrictor regulation of mouse colonic submucosal arterioles is altered in experimental colitis. J Physiol 583: 719-730.
17. Chial HJ, Camilleri M, Ferber I, Delgado-Aros S, Burton D et al. (2003) Effects of venlafaxine, buspirone, and placebo on colonic sensorimotor functions in healthy humans. Clin Gastroenterol Hepatol 1: 211-218.

18. Chial HJ, Camilleri M, Burton D, Thomforde G, Olden KW et al. (2003) Selective effects of serotonergic psychoactive agents on gastrointestinal functions in health. Am J Physiol Gastrointest Liver Physiol 284: G130-137.

19. Hassani H, Lucas G, Rozell B, Ernfors P (2005) Attenuation of acute experimental colitis by preventing NPY Y1 receptor signaling. Am J Physio Gastrointest Liver Physiol 288: G550-556.

20. Chandrasekharan B, Bala V, Kolachala VL, Vijay-Kumar M, Jones D et al (2008) Targeted deletion of neuropeptide Y (NPY) modulates experimental colitis. PLoS One 3: e3304

21. Pang XH, Li TK, Xie Q, He FQ, Cui de J et al. (2010) Amelioration of dextran sulfate sodium-induced colitis by neuropeptide $Y$ antisense oligodeoxynucleotide. Int J Colorectal Dis 25: 1047-1053

22. Petersson J, Schreiber O, Steege A, Patzak A, Hellsten A et al. (2007) eNOS involved in colitis-induced mucosal blood flow increase. Am J Physio Gastrointest Liver Physiol 293: G1281-1287.

23. Sklyarov AY, Panasyuk NB, Fomenko IS (2011) Role of nitric oxide-synthase and cyclooxygenase/lipooxygenase systems in development of experimental ulcerative colitis. J Physiol Pharmacol 62: 65-73.

24. Botta M, Distrutti E, Mencarelli A, Parlato MC, Raffi F et al. (2008) Antiinflammatory activity of a new class of nitric oxide synthase inhibitors that release nitric oxide. ChemMedChem 3: 1580-1588.

25. Webster EL, Torpy DJ, Elenkov IJ, Chrousos GP (1998) Corticotropin-releasing hormone and inflammation. Ann N Y Acad Sci 840: 21-32.

26. Im E, Rhee SH, Park YS, Fiocchi C, Tache Y et al. (2010) Corticotropinreleasing hormone family of peptides regulates intestinal angiogenesis. Gastroenterology 138: 2457-2467.

27. Fukudo S (2007) Role of corticotropin-releasing hormone in irritable bowe syndrome and intestinal inflammation. J Gastroenterol 17: 48-51.

28. Saito-Nakaya K, Hasegawa R, Nagura Y, Ito H, Fukudo S (2008) Corticotropinreleasing hormone receptor 1 antagonist blocks colonic hypersensitivity induced by a combination of inflammation and repetitive colorectal distension. Neurogastroenterol Motil 20: 1147-1156

29. Porcher C, Sinniger V, Juhem A, Mouchet P, Bonaz B (2004) Neuronal activity and CRF receptor gene transcription in the brains of rats with colitis. Am J Physiol Gastrointest Liver Physiol 287: G803-814.

30. Greenwood-Van Meerveld B, Johnson AC, Schulkin J, Myers DA (2006) Long term expression of corticotropin-releasing factor (CRF) in the paraventricula nucleus of the hypothalamus in response to an acute colonic inflammation. Brain Res1071: 91-96.

31. Gay J, Kokkotou E, O'Brien M, Pothoulakis C, Karalis KP (2008) Corticotropinreleasing hormone deficiency is associated with reduced local inflammation in a mouse model of experimental colitis. Endocrinology 149: 3403-3409.

32. Vicario M, Alonso C, Guilarte M, Serra J, Martinez C et al. (2011) Chronic psychosocial stress induces reversible mitochondrial damage and corticotropinreleasing factor receptor type-1 upregulation in the rat intestine and IBS-like gut dysfunction. Psychoneuroendocrinology.

33. Chang L, Sundaresh S, Elliott J, Anton PA, Baldi P et al. (2009) Dysregulation of the hypothalamic-pituitary-adrenal (HPA) axis in irritable bowel syndrome. Neurogastroenterol Motil 21: 149-159.

34. Skobowiat C, Dowdy JC, Sayre RM, Tuckey RC, Slominski AT (2011) Cutaneous hypothalamic-pituitary-adrenal axis homolog: regulation by ultraviolet radiation. Am J Physiol Endocrinol Metab 301: E484-493.

35. Raza K, Hardy R, Cooper MS (2010) The 11beta-hydroxysteroid dehydrogenase enzymes--arbiters of the effects of glucocorticoids in synovium and bone. Rheumatology (Oxford) 49: 2016-2023.

36. Shi XZ, Sarna SK (2009) Homeostatic and therapeutic roles of VIP in smooth muscle function: myo-neuroimmune interactions. Am J Physiol Gastrointest Liver Physiol 297: G716-725.

37. Yukawa T, Oshitani N, Yamagami H, Watanabe K, Higuchi K et al. (2007) Differential expression of vasoactive intestinal peptide receptor 1 expression in inflammatory bowel disease. Int J Mol Med 20: 161-167. 
Citation: Skobowiat C (2011) Contribution of Neuropeptides and Neurotransmitters in colitis. J Veterinar Sci Technol S5:001. doi:10.4172/2157-7579. S5-001

38. Yadav M, Huang MC, Goetzl EJ (2011) VPAC1 (vasoactive intestinal peptide (VIP) receptor type 1) G protein-coupled receptor mediation of VIP enhancement of murine experimental colitis. Cell Immunol 267: 124-132.

39. Gonkowski S, Burlinski P, Skobowiat C, Majewski M, Calka J (2010) Inflammation- and axotomy-induced changes in galanin-like immunoreactive (GAL-LI) nerve structures in the porcine descending colon. Acta Vet Hung 58: 91-103.

40. Talero E, Sanchez-Fidalgo S, Ramon Calvo J, Motilva V (2006) Galanin in the trinitrobenzene sulfonic acid rat model of experimental colitis. Int Immunopharmacol 6: 1404-1412.

41. Talero E, Sanchez-Fidalgo S, Calvo JR, Motilva V (2007) Chronic administration of galanin attenuates the TNBS-induced colitis in rats. Regul Pept 141: 96-104.

42. Ghia JE, Li N, Wang H, Collins M, Deng Y et al. (2009) Serotonin has a key role in pathogenesis of experimental colitis. Gastroenterology 137: 1649-1660.

43. Bertrand PP, Barajas-Espinosa A, Neshat S, Bertrand RL, Lomax AE (2010) Analysis of real-time serotonin (5-HT) availability during experimental colitis in mouse. Am J Physiol Gastrointest Liver Physiol 298: G446-455.

44. Mousavizadeh K, Rahimian R, Fakhfouri G, Aslani FS, Ghafourifar P (2009) Anti-inflammatory effects of 5-HT receptor antagonist, tropisetron on experimental colitis in rats. Eur J Clin Invest 39: 375-383.

45. Ekblad E, Kuhar M, Wierup N, Sundler F (2003) Cocaine- and amphetamineregulated transcript: distribution and function in rat gastrointestinal tract. Neurogastroenterol Motil 15: 545-557.

46. Gunnarsdottir A, Wierup N, Larsson LT, Kuhar MJ, Ekblad E (2007) CARTpeptide immunoreactivity in enteric nerves in patients with Hirschsprung's disease. Eur J Pediatr Surg 17:184-189.
47. Ekblad E (2006) CART in the enteric nervous system. Peptides 27: 2024-2030.

48. Gonkowski S, Burlinski P, Skobowiat C, Majewski M, Arciszewski MB et al (2009) Distribution of cocaine- and amphetamine-regulated transcript-like immunoreactive (CART-LI) nerve structures in the porcine large intestine. Acta Vet Hung 57: 509-520.

49. Tebbe JJ, Ortmann E, Schumacher K, Monnikes H, Kobelt P et al. (2004) Cocaine- and amphetamine-regulated transcript stimulates colonic motility via central CRF receptor activation and peripheral cholinergic pathways in fed, conscious rats. Neurogastroenterol Motil 16: 489-496.

50. Kumar A, Auron M, Aneja A, Mohr F, Jain A et al. (2011) Inflammatory bowe disease: perioperative pharmacological considerations. Mayo Clin Proc 86 748-757.

51. Singh UP, Singh NP, Singh B, Mishra MK, Nagarkatti M et al. (2010) Stem cells as potential therapeutic targets for inflammatory bowel disease. Front Biosci (Schol Ed) 2: 993-1008.

52. Rijnierse A, van Zijl KM, Koster AS, Nijkamp FP, Kraneveld AD (2006) Beneficial effect of tachykinin NK1 receptor antagonism in the development of hapten-induced colitis in mice. Eur J Pharmacol 548:150-157.

53. Cutrufo C, Evangelista S, Cirillo R, Ciucci A, Conte B et al. (2000) Protective effect of the tachykinin NK2 receptor antagonist nepadutant in acute rectocolitis induced by diluted acetic acid in guinea-pigs. Neuropeptides 34: 355-359.

54. Kazi HA, Qian Z (2009) Crocetin reduces TNBS-induced experimental colitis in mice by down regulation of NFkB. Saudi J Gastroenterol 15: 181-187.

55. Wojtkiewicz J, Rowniak M, Crayton R, Barczewska M et al. (2011) InflammationInduced Changes in the Chemical Coding Pattern of Colon-Projecting Neurons in the Inferior Mesenteric Ganglia of the Pig. J Mol Neurosci.
This article was originally published in a special issue, Veterinary Anatomic Pathology handled by Editor(s). Dr. Serkan Erdogan, Dicle University, Turkey; Dr. Bahramsoltani Mahtab, University of leipzig, Germany; Dr. Fawzy Elnady, Cairo University, Egypt 